\title{
CLONAJE Y CARACTERIZACIÓN MOLECULAR IN SILICO DE UN TRANSCRITO DE FOSFOLIPASA A 2 AISLADO DEL VENENO DE LA SERPIENTE PERUANA Lachesis muta
}

\begin{abstract}
Karim L. Jimenez ${ }^{1, a}$, Amparo I. Zavaleta ${ }^{1, b}, V_{\text {Victor Izaguirre }}^{1, b}$, Armando Yarleque $^{2, c}$, Rosio R. Inga ${ }^{2, d}$ RESUMEN

Objetivo. Aislar y caracterizar in silico un transcrito del gen de fosfolipasa $\mathrm{A}_{2}\left(\mathrm{PLA}_{2}\right)$ aislado del veneno de Lachesis muta de la Amazonía peruana. Materiales y métodos. Se amplificó el transcrito del gen SPLA ${ }_{2}$ mediante la técnica de RT-PCR a partir de RNA total utilizando cebadores específicos, el producto de DNA amplificado se insertó en el vector pGEM para su posterior secuenciación. Mediante análisis bioinformático de la secuencia nucleotídica se determinó un marco de lectura abierta de 414 nucleótidos que codifica 138 aminoácidos, incluyendo16 aminoácidos del péptido señal, el peso molecular y el pl fueron de $13976 \mathrm{kDa}$ y 5,66 respectivamente. Resultados. La secuencia aminoacídica denominada Lm-PLA ${ }_{2}$ Perú, contiene Asp49, así como Tyr-28, Gly-30, Gly-32, His-48, Tyr52, Asp99 importantes para la actividad enzimática. La comparación de Lm-PLA - -Perú con las secuencias aminoacídicas de los bancos de datos mostró $93 \%$ de similitud con las $\mathrm{sPLA}_{2}$ de Lachesis stenophrys y más del $80 \%$ con otras $\mathrm{SPLA}_{2}$ de venenos de la familia Viperidae. El análisis filogenético de la secuencia nucleotídica del transcrito del gen sPLA indica que Lm-PLA - -Perú se agrupa con otras sPLA ${ }_{2}\left[\mathrm{Asp}^{49}\right]$ ácidas previamente aisladas del veneno de Bothriechis schlegelii con un $89 \%$ de identidad. El modelaje tridimensional de Lm-PLA 2 -Perú, presenta una estructura característica de $\mathrm{SPLA}_{2}$ del Grupo II formada por tres hélices- $\alpha$, una lámina- $\beta$, una hélice corta y un lazo de unión con calcio. Conclusión. La secuencia nucleotídica corresponde al primer transcripto del gen de $\mathrm{PLA}_{2}$ clonado a partir del veneno de la serpiente Lachesis muta, que habita en la selva del Perú.
\end{abstract}

Palabras clave: Fosfolipasas $A_{2}$; Lachesis muta; Venenos de serpiente; Clonación molecular; Biología computacional (fuente: DeCS BIREME).

\section{MOLECULAR CLONING AND CHARACTERIZATION IN SILICO OF PHOSPHOLIPASE A 2 TRANSCRIPTO ISOLATED FROM Lachesis muta PERUVIAN SNAKE VENOM}

\begin{abstract}
Objective. Isolate and characterize in silico gene phospholipase $A_{2}\left(P_{2} A_{2}\right)$ isolated from Lachesis muta venom of the Peruvian Amazon. Material and methods. Technique RT-PCR from total RNA was using specific primers, the amplified DNA product was inserted into the pGEM vector for subsequent sequencing. By bioinformatic analysis identified an open reading frame of 414 nucleotides that encoded 138 amino acids including a signal peptide of 16 aminoacids, molecular weight and pl were $13976 \mathrm{kDa}$ and 5.66 respectively. Results. The aminoacid sequence was called Lm-PLA ${ }_{2}-P e r u$, contains an aspartate at position 49, this aminoacid in conjunction with other conserved residues such as Tyr-28, Gly-30, Gly-32, His-48, Tyr52, Asp99 are important for enzymatic activity. The comparison with the amino acid sequence data banks showed of similarity between $\mathrm{PLA}_{2}$ from Lachesis stenophrys (93\%) and other $\mathrm{PLA}_{2}$ snake venoms and over $80 \%$ of other SPLA family Viperidae venoms. A phylogenetic analysis showed that Lm-PLA - Peru grouped with other acidic $\left[\mathrm{Asp}^{49}\right] \mathrm{sPLA}_{2}$ previously isolated from Bothriechis schlegelii venom showing $89 \%$ nucleotide sequence identity. Finally, the computer modeling indicated that enzyme had the characteristic structure of sPLA ${ }_{2}$ group II that consisted of three $\alpha$-helices, a $\beta$-wing, a short helix and a calcium-binding loop. Conclusion. The nucleotide sequence corresponding to the first transcript of gene from PLA ${ }_{2}$ cloned of Lachesis muta venom, snake from the Peruvian rainforest.
\end{abstract}

Key words: Phospholipases $A_{2}$; Lachesis muta; Snake venoms; Cloning, molecular; Computational biology (source: MeSH NLM).

\section{INTRODUCCIÓN}

Los venenos de serpientes presentan una gran diversidad de acciones biológicas y composición química compleja, constituyen bibliotecas de compuestos bioactivos con invalorable potencial terapéutico, lo cual motiva continuas investigaciones para convertir estas sustancias tóxicas en modelos para el desarrollo de otras de interés terapéutico y biotecnológico. Los venenos pueden contener uno o más compuestos tóxicos en diferentes

Laboratorio de Biología Molecular, Facultad de Farmacia y Bioquímica, Universidad Nacional Mayor de San Marcos, Lima, Perú

2 Laboratorio de Biología Molecular, Facultades de Ciencias Biológicas, Universidad Nacional Mayor de San Marcos, Lima, Perú.

a Química Farmacéutica, Magíster en Biotecnología; b Químico Farmacéutico, Doctor; ' Doctor en Ciencias Biológicas; d Bióloga con Mención en Biología Celular y Genética. 
proporciones dependiendo de la familia, género o especie de serpiente, por lo que causan síntomas clínicos característicos del espécimen.

Por otro lado, los venenos de las serpientes constituyen un serio problema de salud pública; en 1998, Chippaux analizó la situación mundial, basado principalmente en los registros hospitalarios y las estadísticas de las autoridades sanitarias y reportó que el número total de mordeduras de serpiente supera los cinco millones con una tasa de mortalidad por mordedura de serpiente a nivel mundial de 125000 por año ${ }^{(1)}$.

En el Perú, las serpientes venenosas endémicas están representadas por los géneros Bothrops y Lachesis (2). La Lachesis muta, tradicionalmente conocida como "shushupe" o "bushmaster" habita en diversas regiones de la selva amazónica, su veneno presenta actividades procoagulantes, proteolíticas y de fosfolipasa $A_{2}\left(P L A_{2}\right)$ de mayor magnitud que las especies del género $B o-$ throps ${ }^{(3)}$. La mordedura accidental por L. muta causa dolor local, edema, hemorragia, necrosis y desórdenes sistémicos como naúseas, vómitos, diarrea, hipotensión, bradicardia, altera la coagulación y la función renal ${ }^{(4)}$. Las fosfolipasas $A_{2}$ han demostrado gran variabilidad de acciones biológicas dependiendo del tipo de serpiente del cual provengan, así se han descrito neurotóxicas, miotóxicas, anticoagulantes, cardiotóxicas o hemolíticas ${ }^{(4)}$.

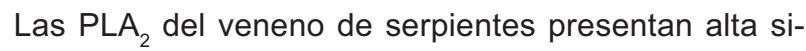
militud estructural con fosfolipasas de los mamíferos pero difieren considerablemente en los efectos farmacológicos que producen. La purificación y caracterización bioquímica de estas enzimas es difícil, por la gran diversidad de isoformas existentes en la glándula salival ofídica y por la dificultad de obtener el veneno de la fuente natural. Por tanto, el clonaje de los genes a partir del RNA total es una metodología alternativa para la obtención de cantidades adecuadas de estas proteínas y su posterior caracterización, destacando el hecho de que no es necesario sacrificar al espécimen en estudio.

En los últimos años se ha incrementado la caracterización y simulación de proteínas a partir de la secuencia nucleotídica del gen de interés mediante el uso de programas bioinformáticos de libre acceso, este tipo de simulaciones de estructuras complejas por medio de las computadoras es denominado modelaje in silico.

Así, en este estudio se presenta la secuencia del transcrito de un gen de $\mathrm{PLA}_{2}$ de $L$. muta proveniente de la Amazonía peruana, y se compara con las isoformas encontradas en la ponzoña de $L$. muta de Brasil y de otras especies relacionadas, mediante el clonaje del DNAc amplificado, secuenciación nucleotídica, análisis bioinformático y modelaje estructural in silico de la proteína.

\section{MATERIALES Y MÉTODOS}

\section{SÍNTESIS DE DNAC}

Se obtuvieron $400 \mu \mathrm{L}$ del veneno crudo de una serpiente adulta de Lachesis muta procedente de Pucallpa, región Ucayali, mantenida en el Serpentario Oswaldo Meneses del Museo de Historia Natural de la Universidad Nacional Mayor de San Marcos. Del veneno recién extraído se aisló y purificó el RNA total usando el kit Total RNA Purification (AMRESCO), de acuerdo con las instrucciones del fabricante, al RNA total se añadió DNasa e inhibidor de RNasa y se incubó a $37^{\circ} \mathrm{C}$ durante $30 \mathrm{~min}$. Después, se añadió el tampón de reacción, hexanucleotídicos $20 \mu \mathrm{M}$, ditiotreitol $0,1 \mathrm{M}$, desoxinucleótidos 200 $\mu \mathrm{M}$ y transcriptasa inversa MMLV 200U. La mezcla de reacción se incubó a $37^{\circ} \mathrm{C}$ por una hora.

\section{AMPLIFICACIÓN DE GENES SPLA $A_{2}$}

Aproximadamente $50 \mathrm{ng}$ de DNAc se amplificó mediante PCR en un volumen final de $25 \mu \mathrm{L}$. La mezcla de reacción contenía: $\mathrm{KCl} 50 \mathrm{mM}$, Tris/ $\mathrm{HCl}(\mathrm{pH} 9$ a 25 $\left.{ }^{\circ} \mathrm{C}\right) 10 \mathrm{mM}$, triton X-100 0,1\% (v/v), $\mathrm{MgCl}_{2} 1,5 \mathrm{mM}$, de cada desoxinucleótido $200 \mu \mathrm{M}$, cebadores 25 pmoles descritos por Lisano et al. (5), y Taq DNA polimerasa $1,5 \mathrm{U}$. Las condiciones de cada ciclo de reacción programadas en un termociclador (Perkin Elmer 2400) fueron: desnaturalización a $94^{\circ} \mathrm{C}$ por $45 \mathrm{~s}$, hibridación a $55^{\circ} \mathrm{C}$ por 1 min y polimerización a $72{ }^{\circ} \mathrm{C}$ por $2 \mathrm{~min}$. Estas condiciones se repitieron durante 35 ciclos y al final se incubó a $72{ }^{\circ} \mathrm{C}$ por $7 \mathrm{~min}$. En todos los casos se consideró controles negativos de la reacción de PCR sin añadir DNA molde. El producto amplificado se separó por electroforesis en gel de agarosa al $1 \%$ en buffer TAE 1X a $80 \mathrm{~V}$; posteriormente, se coloreó con bromuro de etidio y el fragmento de DNA amplificado de aproximadamente 450 pb se purificó con el kit Cyclo-Pure-Gel Extraction (AMRESCO) según instrucciones del fabricante.

\section{CLONAJE DEL DNAC AMPLIFICADO}

Para la ligación se utilizó el sistema de clonaje $p G E M \circledR-T$ Easy (Promega) según las instrucciones del fabricante y se incubó a $4{ }^{\circ} \mathrm{C}$ durante 12 horas. Células competentes de $E$. coli $\mathrm{DH}-5 \alpha$ se transformaron con $5 \mu \mathrm{L}$ del producto de ligación y se sembraron en agar LB conteniendo ampicilina/IPTG/X-Gal a $37^{\circ} \mathrm{C}$ durante 24 horas. 


\section{PURIFICACIÓN DEL DNA PLASMÍDICO RECOMBINADO}

Las colonias de color blanco se crecieron en $10 \mathrm{~mL}$ de caldo LB con ampicilina $75 \mu \mathrm{g} / \mathrm{mL}$ a $37^{\circ} \mathrm{C}, 180 \mathrm{rpm}$ durante 24 horas. Para la extracción y la purificación de los plásmidos recombinantes se utilizó el kit Cyclo-Prep ${ }^{\mathrm{TM}}$ Plasmid DNA Purification (AMRESCO) siguiendo las instrucciones del fabricante. La concentración del DNA plasmídico se determinó mediante espectrofotometría a $260 \mathrm{~nm}$.

\section{SECUENCIACIÓN Y ANÁLISIS}

La secuenciación de los clones obtenidos se realizó utilizando los cebadores T7 y SP6 pertenecientes al vector $\mathrm{PGEM} \AA$-T en el secuenciador $\mathrm{ABI} 3100$ (Applied Biosystems) de la Universidad Peruana Cayetano Heredia. Para el análisis de las secuencias clonadas se utilizaron diferentes programas bioinformáticos de libre acceso.

\section{RESULTADOS}

\section{AMPLIFICACIÓN, CLONAJE Y SECUENCIACIÓN DE GENES $s P L A_{2}$}

Utilizando la RT-PCR se amplificó un fragmento de DNA de aproximadamente de 448 pares de bases a partir del RNA total obtenido de Lachesis muta (Figura 1). El producto amplificado se clonó en el vector $\mathrm{PGEM} 囚-T$ y se obtuvieron 22 clones, seis de los cuales se secuenciaron por ambos lados, sin obtener redundancias, lo que indica

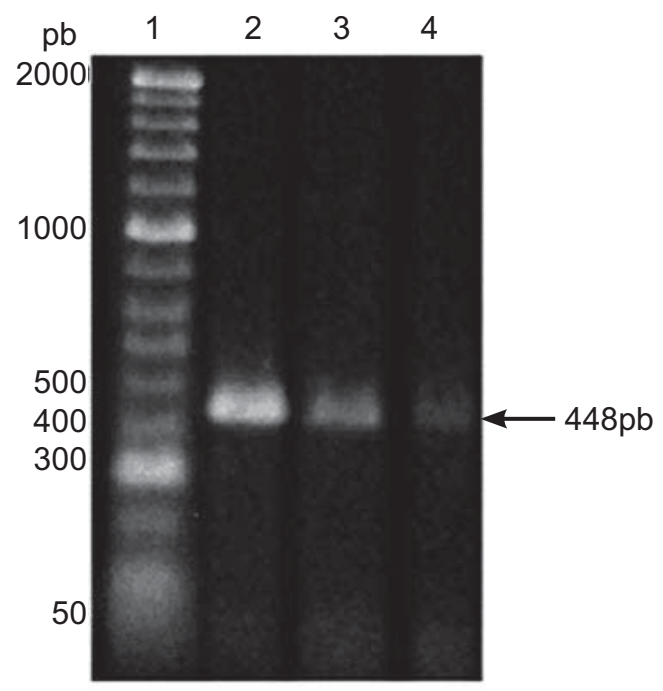

Figura 1. Amplificación de sPLA 2 a partir del DNAc obtenido de la glándula salival de L. muta. Línea (muestra): 1, Hyperladder II; 2-4, producto de la amplificación de sPLA a partir de 50, 25 y $5 \mathrm{ng}$ de DNAc respectivamente. que el fragmento de DNA amplificado corresponde a un único producto. La secuencia nucleotídica del transcrito del gen sPLA ${ }_{2}$ obtenida se depositó en el Gene Bank, el código de acceso asignado es GQ265920.

\section{ANÁLISIS BIOINFORMÁTICO DE LA SECUENCIA NUCLEOTÍDICA}

La secuencia nucleotídica del transcrito del gen $P L A_{2}$ analizada mediante el programa Blastn (http://www. ncbi.nlm.nih.gov/) mostró más de $80 \%$ de identidad con $\mathrm{sPLA}_{2}$ ácidas de serpientes de la familia Viperidae (Tabla 1). Las secuencias del Gene Bank que presentaron mayor similitud con GQ265920, se alinearon empleando el Clustal W (http://www.ebi.ac.uk/Tools/clustalw2/index. html). En el alineamiento se observó que las regiones no traducidas 5' y 3' son más conservadas que las regiones codificantes, precisamente los cebadores FPLA $_{2}$ y $\mathrm{RPLA}_{2}$ utilizados en la RT-PCR están diseñados a partir de las regiones altamente conservadas, siendo útiles para la amplificación y el clonaje de transcritos génicos de $\mathrm{SPLA}_{2}$ de otras serpientes de la familia Viperidae. Además, se observó un mayor número de substituciones a nivel de la primera y segunda posición nucleotídica de cada codón, esta variabilidad podría explicar la diversidad de isoenzimas de $\mathrm{SPLA}_{2}$ con actividades biológicas diferentes, característica de estas proteínas provenientes de serpientes.

Empleando el alineamiento obtenido por el Clustal W, en formato ( ${ }^{*}$.phy) y el método basado en caracteres de máxima probabilidad del programa Phylip (http://evolution.genetics.washington.edu/phylip.html) se elaboró el árbol filogenético con las ramificaciones más probables en función de los cambios en la secuencia de DNA. Se usó la secuencia nucleotídica incluyendo la zona correspondiente al péptido señal. Al ser comparada con otras $\mathrm{SPLA}_{2}$ similares de la familia Viperidae, cuyos números de acceso se describe en la Tabla 1 , se evidenció que

Tabla 1. Comparación del porcentaje de identidad (I) de la secuencia nucleotídica del transcrito del gen $\mathrm{SPLA}_{2}$ de L. muta con las de otras serpientes de la familia Viperidae.

\begin{tabular}{llccl}
\hline Proteína & \multicolumn{1}{c}{ Organismo } & $\begin{array}{c}\text { Valor } \\
\text { de e }\end{array}$ & $\begin{array}{c}\text { I } \\
(\%)\end{array}$ & Acceso \\
\hline cTgPLA $_{2}{ }^{-1}$ & Trimeresurus gramineus & $1 \mathrm{e}^{-163}$ & 90 & $\mathrm{D} 31774.1$ \\
\hline Ts-A1 & Trimeresurus stejnegeri & $5 \mathrm{e}^{-162}$ & 89 & $\mathrm{AY} 211939.1$ \\
$\mathrm{PLA}_{2}$ ácida & Bothriechis schlegelii & $1 \mathrm{e}^{-158}$ & 89 & $\mathrm{AY} 764137.1$ \\
$\mathrm{PLA}_{2}$ ácida & Trimeresurus gracilis & $6 \mathrm{e}^{-146}$ & 88 & $\mathrm{AY764141.1}$ \\
$\mathrm{PLA}_{2}$ ácida & Crotalus viridis viridis & $2 \mathrm{e}^{-135}$ & 86 & $\mathrm{AY} 120875.1$ \\
$\mathrm{PLA}_{2}$ & Trimeresurus okinavensis & $7 \mathrm{e}^{-126}$ & 85 & $\mathrm{D} 49388.1$ \\
$\mathrm{PLA}_{2}$ & Sistrurus catenatus tergeminus & $2 \mathrm{e}^{-125}$ & 85 & $\mathrm{AY} 508692.1$ \\
$\mathrm{PLA}_{2}$ ácida & Trimeresurus borneensis & $2 \mathrm{e}^{-125}$ & 85 & $\mathrm{AY} 355178.1$ \\
$\mathrm{PLA}_{2}$ & Bothrops insularis & $1 \mathrm{e}^{-123}$ & 84 & $\mathrm{AF} 490535.1$ \\
\hline
\end{tabular}


Phylogenetic tree

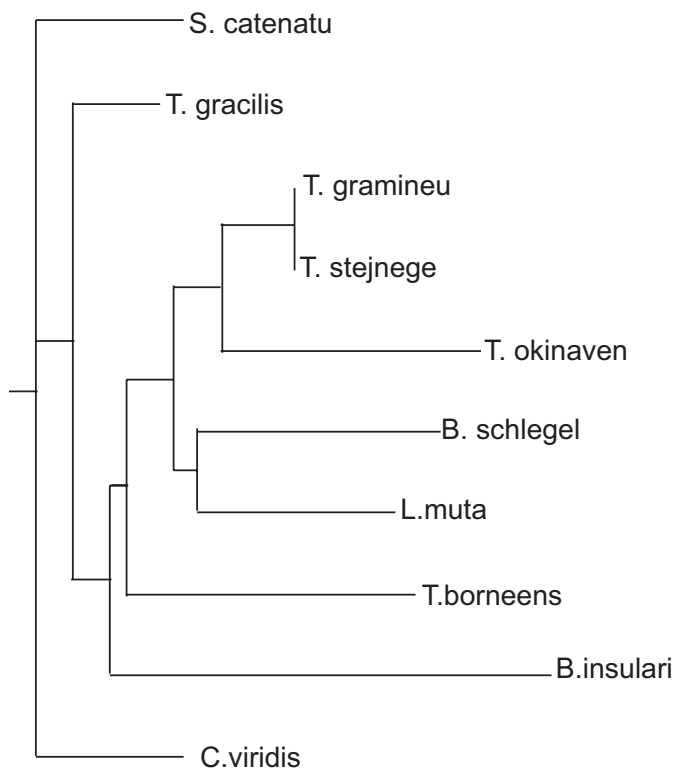

Figura 2. Árbol filogenético de la secuencia nucleotídica GQ265920 de L. muta comparada con otras SPLA ${ }_{2}$ de serpientes de la familia Viperidae elaborado con el programa Phylip.

GQ265920 esta evolutivamente más relacionada con una $\mathrm{SPLA}_{2}$ ácida, aislada del veneno de Bothriechis schlegelii, al presentar $89 \%$ de identidad de secuencia (Figura 2).

\section{ANÁLISIS BIOINFORMÁTICO DE LA SECUENCIA AMINOACÍDICA}

La secuencia nucleotídica del transcrito del gen de $\mathrm{sPLA}{ }_{2}$ fue traducida utilizando el programa Translate tool (http:// ca.expasy.org), se obtuvo una secuencia de 138 aminoácidos. La secuencia aminoacídica denominada $\mathrm{Lm}^{-P \mathrm{PA}_{2}-}$ Perú se comparó con otras reportadas en los bancos de datos a través del programa Blastp. Esta secuencia presentó similitud con la fosfolipasa secretora $\mathrm{A}_{2}$ de Lachesis stenophrys con $93 \%$ y más del $80 \%$ con otras secuencias de $\mathrm{SPLA}_{2}$ de serpientes de la familia Viperidae. Las secuencias aminoacídicas que mostraron mayor similitud con Lm-PLA 2 -Perú se alinearon con el programa Clustal $W$. En la Figura 3 se presentan los aminoácidos conservados descritos con asteriscos, los que conforman el sitio de unión al calcio y el centro activo así como los residuos Cys que forman los puentes disulfuro.

Por otro lado, a partir de la secuencia aminoacídica madura se determinó el peso molecular en 13976 $\mathrm{kDa}$ y el punto isoeléctrico 5,66 mediante el programa Protparam (http://ca.expasy.org/tools/protparam.html). Con el programa SWISS MODEL (http://www.expasy. org/swissmod/SWISS-MODEL.html)(6) se empleó modelos tridimensionales reales de proteínas similares obtenidas por cristalización el cual nos permitió elucidar la estructura de $L m-P L A_{2}-P e r u ́$. Para ello, en primer lugar se realizó una comparación de la secuencia, la cual mostró el $76,61 \%$ de identidad tridimensional con la proteína monomérica de SPLA 2 -Asp 49 del veneno de Agkistridon piscivorus ${ }^{(7)}$.

A partir de de la estructura tridimensional de mayor similitud seleccionada se propuso un modelo teórico de la proteína Lm-PLA ${ }_{2}$-Perú, el cual se presenta en la Figura 4 , en donde se observa la estructura secundaria formada por tres hélices- $\alpha$, una lámina- $\beta$, una hélice corta y un lazo de unión con calcio. La estructura terciaria contiene un canal hidrofóbico en la porción amino terminal donde se ubica el centro catalítico. Además, presenta la forma elipsoide aplanada de dimensiones $45 \times 30 \times 20 \AA$, que se estabiliza por la presencia de siete enlaces disulfuro altamente conservados, este patrón estructural es característico de las $\mathrm{SPLA}{ }_{2}$ del grupo II.

El conocimiento de la estructura tridimensional es crucial para entender la función de una proteína y para algunas aplicaciones como el diseño de nuevas drogas. Las proteínas homólogas son aquellas que comparten alto grado similitud estructural y, por ende, la configuración espacial. Este tipo de proteínas con el transcurso del tiempo, después de eventos de transferencia horizontal o vertical, acumularon cambios en las secuencias de los genes resultantes y, por tanto, en las secuencias de las proteínas. Sin embargo, en las proteínas homólogas, la estructura espacial o plegamiento de la proteína se conserva a pesar de cambios en su estructura primaria. La estructura tridimensional de las SPLA ${ }_{2}$ de clase II es muy conservada, de este modo se infiere que la enzima es catalíticamente activa, este análisis es importante para planificar una metodología de expresión de la proteína. Por ejemplo, podriamos utilizar la medición de la actividad catalítica para permitir determinar la eficiencia de la expresión de la proteína.

\section{DISCUSIÓN}

Las $\mathrm{SPLA}_{2}$ del veneno de serpientes son enzimas que alcanzaron la máxima diversidad evolutiva, muestran aparente similitud estructural y difieren considerablemente en los efectos farmacológicos que producen ${ }^{(8-11)}$. Estas $\mathrm{sPLA}_{2}$ no solamente constituyen un excelente material de estudio, sino también poseen gran potencial farmacológico para el diseño de nuevas drogas, elucidación de los diferentes mecanismos de acción y tratamiento de diversas enfermedades en las que están implicadas ${ }^{(12-16)}$. Sin embargo, la diversidad de isoenzimas de $\mathrm{SPLA}_{2}$ en los venenos de serpientes dificulta la purificación y caracterización por métodos bioquímicos convencionales ${ }^{(17)}$. 
I. muta-Peru

L.stenophrys

B. schlegeli i

T.gramineus

T.stejnegeri

B.erythromelas

T.flavoviridis

S.miliarius

S. catenatus

C.viridis

T.gracilis

L.muta-Peru

L.stenophrys

B. schlegelii

T.gramineus

T.stejnegeri

B. erythromelas

T. flavoviridis

S.miliarius

S.catenatus

C. viridis

T.gracilis

L.muta-Peru

L.stenophrys

B.schlegelii

T.gramineus

T.stejnegeri

B. erythromelas

T. flavoviridis

S.miliarius

S.catenatus

C.viridis

T.gracilis
HLLQFGDLINKIARRN - GILYYSFYGCYCGLGGRGRPQDATDR CCFVHDCCYGKVTGCNPK HLLQFGDLIDKIAGRS - GFWYYGFYGCYCGLGGRGR PQDATDRCCFVHD CCYGKVTGCDPK DLLQFEGMIMTIAGRS - GIWYYGSYGCYCGAGGQGKPQDASDRCCFVHDCCYGKVTECNPK HLMQFETLIMKVAGRS - GVWYYGSYGCFCGAGGQGRPQDASDR CCFVHDCCYGKVNGCDPK H LMQFETLIMKVAGRS - GVWYYGSYGCFCGAGGQGRPQDASDR C CFVHDCCYGKVNGCDPK SLVQFETLIMKIAGRS - GVWYYGSYGCYCGSGGQGRPQDASDRCCFVHDCCYGKVTDCDPK HLMQFENMI KKVTGRS - GIWWYGSYGCYCGKGGEGR PQDP SDRCCFVHDCCYGKVTGCDPK HLIQFETLIMKIAGRS - GVFWYSAYGCYCGWGGYGRPODATDRCCFVHDCCYGKVTGCNPK HLIQFETLIMKIAGRN - GVFWYSAYGCYCGWGGYARPQDTSDRCCFVHDCCYGKVTGCNPK S LVOF EML IMKVAKRS - GLF SYSAYGCYCGWGGHGR PODATDHCCFVHDCCYGKVTDCNPK S LMQFEML I M KLAKS S - GMFWYSAYGCYCGWGGQGRPQDATDRCCFVHDCCYGKATGCDPK

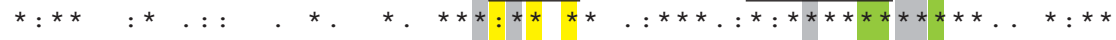

NDIYTYSEENGA IVCGGDNPCKKEI CECDRDAA I CFRDNLDTYDNK - YWFLPRKYCKEES KDIYTYSEENGA IVCGGDNPCKKEI CECDRDAA I CFRDNLDTYDNK-YWLFPNKYCKEES KDSYTYTEENGAVVCGGDDP CMKEVCECDKNAA I CFRDNLDTYDNK - YWFYPAKYCOEES KDFYTYSEENGA IVCGGDDPCKKEI CECDKDAA I CFRDNKDTYDNK-YWFFPAKNCQEES KDFYTYSEENGA IVCGGDDP CKKEI CECDKDAA I CFRDNKDTYDNK-YWFFPAKNCQEES ADVYTYSEENGVVVCGGDDPCKKQI CECDRVAATCFRDNKDTYDNK - YWFFPAKNCQEES DDFYIYSSENGDIVCGDDDLCKKEVCECDKAAA I CFRDNMDTYQNK - YWFYPASNCKEES KDFYSYTEENGE IVCGGDDPCKKO I CECDRVAA I CFRDN I PTYDDK - YWRFPTENCQEEP KDFYSYSEENGEIVCGGDDP CKKQI CECDRVAA I CFRDN I PTYDDK - YWRF PTENCQEEP TASYTYSEENGE IVCGGDD P CKKQVCECDRVAA I CFRDNI PTYDNK - YWRF P PENCQEEP KDVYTYSEENGDIVCGGDDPCRKEVCECDKAAA I CFRDNMDTYNSKTYWMFPAKNCOEES

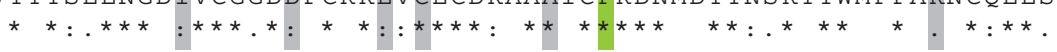

EPC 122

EPC 122

EPC 122

EPC 122

EPC 122

EPC 122

EPC 122

EPC 122

EPC 122

EPC 122

EPC 123

***

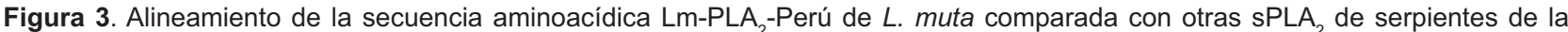
familia Viperidae utilizando el programa Clustal W. Se representa en amarillo el sitio de unión al $\mathrm{Ca}^{++}$, en verde los aminoácidos conservados que conforman el centro activo y en gris los 14 residuos Cys que conforman los siete puentes disulfuro.

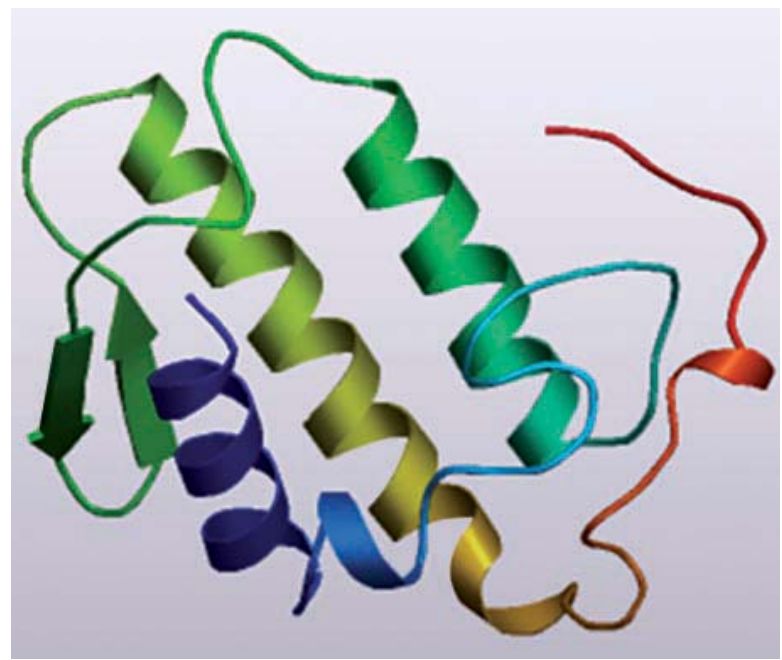

Figura 4. Estructura terciaria teórica de Lm-PLA 2 -Perú, obtenida mediante el servidor SWISS-MODEL.
En este estudio se determinó la secuencia completa del gen $\mathrm{SPLA}_{2}$ a partir del RNAm que codifica la $\mathrm{SPLA}_{2}$ aislada del veneno de Lachesis muta proveniente de la Amazonía peruana, usando para ello las técnicas de RTPCR, clonaje, secuenciación y análisis bioinformático. La secuencia nucleotídica (GQ265920) obtenida codifica la proteína madura de una $\mathrm{SPLA}_{2}$, al respecto solo se han descrito secuencias parciales del amino terminal de algunas $\mathrm{SPLA}_{2}$ de $L$. muta ${ }^{(18-21)}$. La proteína obtenida por análisis bioinformático denominada Lm-PLA 2 -Perú, contiene 138 aminoácidos, que incluye al péptido señal. En base a los aminoácidos altamente conservados se deduce que es una enzima dependiente de $\mathrm{Ca}^{++}$y que pertenece a las $\mathrm{SPLA}_{2}[$ Asp49] del grupo II.

El alineamiento múltiple de la secuencia aminoacídica Lm-PLA $A_{2}$-Perú con otras SPLA $_{2}$ de la familia Viperidae, muestra los aminoácidos altamente conservados tales 
como los 14 residuos de cisteína involucrados en la formación de siete enlaces disulfuro en las posiciones: 27-117, 29-45, 44-96, 50-122, 51-89, 58-82, 76-87; los cuales son necesarios para estabilizar la estructura terciaria de la enzima. La conformación tridimensional se caracteriza por la presencia de dos $\alpha$-hélices largas y antiparalelas que definen el canal hidrofóbico a través del cual se conduce al sustrato fosfolipídico hasta el centro activo (Figura 4). Este último está formado por cuatro residuos clave His48, Asp49, Tyr52 y Asp99, que son importantes para la actividad enzimática. El hidrógeno de His se une a una molécula de agua para la hidrólisis, mientras que el Asp49 coordina y posiciona al $\mathrm{Ca}^{++}$que se une para polarizar tanto al grupo fosfato como al sn-2 carbonilo del fosfolípido durante la hidrólisis. Se ha descrito, que la sustitución del Asp49 por Lys, Arg, Ser, GIn, o Asn entre otros disminuye la afinidad por el calcio, con la consecuente pérdida de la actividad enzimática ${ }^{(22,23)}$. Otra región altamente conservada en las $\mathrm{SPLA}_{2}$ es el lazo de unión al calcio formado por Tyr28, Gly30 y Gly32, el grupo carboxilo de estos aminoácidos en conjunto con dos moléculas de agua completan la esfera de coordinación del calcio, formando una bipirámide pentagonal necesaria para la actividad enzimática ${ }^{(24)}$. El reemplazo de $\mathrm{Ca}^{++}$por iones como $\mathrm{Sr}^{++} \mathrm{u}$ otros cationes divalentes inhiben la actividad enzimática ${ }^{(25,26)}$.

De las secuencias aminoacídicas de $\mathrm{SPLA}_{2}$ homólogas a Lm-PLA $A_{2}$-Perú que se muestran en la Tabla 1 , solo se han realizado estudios de la actividad biológica en Ts-A1 correspondiente a la fosfolipasa de la serpiente Trimeresurus stejnegeri proveniente de Taiwan-China, está enzima presenta 79 y $88 \%$ de identidad y similitud respectivamente con respecto a Lm-PLA 2 -Perú. Ts-A1 es una proteína ácida con un $\mathrm{pl}$ de 4.8 , in vitro presenta actividad catalítica alta en presencia de diversos sustratos y es dependiente de $\mathrm{Ca}^{++}(27)$, estos datos apoyan las características de la actividad catalítica determinada in silico de Lm-PLA 2 -Perú.

Por otro lado, varios estudios relacionan el carácter ácido de $\mathrm{SPLA}_{2}$ del grupo II con ciertas actividades biológicas. Así tenemos: LM-PLA ${ }_{2}-\mathrm{I}$ y II aisladas de Lachesis muta de Brasil ${ }^{(18,28)}$, BE-I-PLA ${ }_{2}$ de Bothrops erythromelas ${ }^{(29)} \mathrm{y}$ Bp-PLA ${ }_{2}$ de Bothrops pauloensis ${ }^{(30)}$. Asimismo, Roberto y col. 2004 elaboraron una genoteca de DNAc de de Bothrops jararacusu, aislando clones conteniendo una $\mathrm{sPLA}_{2}$ ácida cuya secuencia mide $366 \mathrm{pb}$, presentando actividad inductora de edema, efecto inhibitorio de la agregación plaquetaria y actividad hemolítica ${ }^{(31)}$. Por el carácter ácido de Lm-PLA ${ }_{2}$-Perú $(p / 5,66)$ es probable que presente alguna de estas actividades. Al respecto, diversos estudios indican que el carácter ácido es importante para presentar este efecto; pero además se requiere los residuos Glu6, Asp114 o Asp115, Trp20,
Trp21, Tyr113 y Trp119 ${ }^{(32)}$, la substitución de uno o más de estos disminuye la actividad antiagregante plaquetaria. En el caso de Lm-PLA 2 -Perú no contiene estos residuos en las posiciones indicadas, por tanto, no debería presentar dicha actividad, esta hipótesis se debe confirmar mediante la expresión de la enzima y la evaluación de la actividad in vivo.

De todas las secuencias publicadas de Lachesis muta se encontraron seis provenientes de serpientes de diferentes áreas geográficas, estas son: fracción $16^{\left({ }^{33}\right)} y$ fracción 18 de Perú y Bolivia respectivamente, LM-PLA ${ }_{2}-1$ y LM-PLA 2 -II de Brasil (12); y LmTX-I y LmTX-II descritas por Sigma-Aldrich ${ }^{20)}$. Al comparar los 22 aminoácidos del amino terminal de las secuencias antes mencionadas mediante el programa Clustal $W$ (Figura 5 ) se observó que Lm-PLA 2 -Perú presenta mayor identidad con las fracciones 16 y 18 aisladas de especímenes de Perú y Bolivia respectivamente. Con respecto a las $\mathrm{SPLA}_{2}$ provenientes de Brasil y de Sigma Aldrich se observa mayor número de substituciones aminoacídicas; lo que demuestra el alto grado de variabilidad intra-específica de $\mathrm{SPLA}_{2}$ en el veneno de Lachesis muta. Similares resultados se han reportado en Vipera palestinae ${ }^{(34)}$, Bothrops asper ${ }^{(35)}$ y Trimeresurus flavoviridis ${ }^{(36)}$.

Esta variabilidad se ha relacionado con las características ambientales del área geográfica donde habitan y al tipo de dieta disponible ${ }^{(36,37)}$. Las variaciones intra-específicas reflejan la adaptación dinámica y la evolución a la que han sido sometidas las $\mathrm{SPLA}_{2}$ del veneno de serpientes, diversos estudios han descrito que las proteínas de los venenos de serpientes se han adaptado a una evolución Darwiniana positiva y a mutaciones aceleradas. En principio, en la estructura del RNA mensajero de proteínas funcionales se espera encontrar mayor similitud en las zonas codificantes que en las UTRs, en el caso de las $\mathrm{SPLA}_{2}$, sucede lo contrario, en la región codificante existe una baja similitud. Las regiones

\begin{tabular}{|lll|}
\hline Lm-PLA2-Peru & HLLQFGDLINKIARRNGILYYSF & 23 \\
FraCCion-16 & HLLQFGDLINKIARRAISYYG & 22 \\
FraCCIOn-18 & HLLQFEQLIRKIAGRSFRYYG & 22 \\
LM-PLA2-I & HLLQFGDLIDKIAGRSFWYYG & 22 \\
LM-PLA2-II & HLLQFEQLIRKIAGR-GFRYYGF & 22 \\
LM-TX-I & HLLQFNKMIKFETRKNAIPFYAF & 23 \\
LM-TX-II & HLLQFNKMIKFETRKNAIPFYAF & 23 \\
\hline
\end{tabular}

Figura 5. Comparación de la secuencia aminoterminal de Lm$\mathrm{PLA}_{2}$-Perú con las de otras isoenzimas aisladas de Lachesis muta provenientes de diferentes fuentes. Fracción 16 y 18 (Iquitos-Perú y Santa Cruz de la Sierra-Bolivia) (20). LM-PLA2-I, y LM-PLA2-II (Brasil) ${ }^{(18)}$. LmTX-I and LmTX-II (Sigma-Aldrich) ${ }^{(19,21)}$. 
UTR 5' y 3' están más conservadas que las regiones codificantes, presentan una identidad de nucleótidos de 95, 67 y 89\% en las regiones 5'UTR, codificante y 3'UTR respectivamente. En la región que codifica la proteína madura se analizó las sustituciones nucleotídicas en la primera, segunda y tercera posición de los codones y se determinó 32,$1 ; 30,0$ y $28,6 \%$ respectivamente. El alto porcentaje de substituciones nucleotídicas en la primera y segunda posición comparado con la tercera es inusual, en la mayoría de casos la tercera posición es más variable que la primera y segunda posición. Las substituciones en la primera y segunda posición originan numerosas isoenzimas que contienen actividades biológicas diversas ${ }^{\left({ }^{37}-39\right)}$. Las secuencias nucleotídicas que codifican las $\mathrm{SPLA}_{2}$ presentan regiones de alta $\mathrm{y}$ baja variabilidad por lo que podrían ser consideradas como cronómetros moleculares para estudiar las relaciones filogenéticas intraespecíficas, esto permitiría encontrar patrones de secuencias específicas para la identificación de género e incluso especie, ya que la determinación precisa de la especie de serpiente que ocasiona una mordedura accidental es crucial para la elección del suero antiofídico apropiado.

El clonaje y caracterización molecular in silico de una $\mathrm{sPLA}_{2}$ del veneno de $L$. muta abre nuevas alternativas de estudios de otros especímenes de zonas geográficas distintas para confirmar la variabilidad de secuencia aminoacídica, las diversas actividades biológicas que producen, así como encontrar relaciones estructurafunción entre los diversos procesos que desencadenan estas toxinas, asimismo encontrar prototipos moleculares para el diseño de nuevos fármacos para $\mathrm{SPLA}_{2}$. La obtención de sueros antiofídicos más específicos y del mismo modo, el clonaje de las diversas isoenzimas facilita la expresión y el estudio amplio de la proteína.

\section{AGRADECIMIENTOS}

Los autores de esta investigación expresan su agradecimiento al Consejo Superior de Investigaciones (CSI) del Vicerrectorado de Investigaciones de la Universidad Nacional Mayor de San Marcos por haber apoyado financieramente el estudio PEM2003A01 en la modalidad de Proyecto Multidisciplinario. Uno de los autores (K. Jiménez) obtuvo su grado de Magíster en Biotecnología con parte de esta investigación y la subvención 162-2006-CONCYTEC-OAJ.

\section{Financiamiento}

Consejo Superior de Investigaciones, Vicerrectorado de Investigación, Universidad Nacional Mayor de San Marcos.

\section{Conflictos de Interés}

Los autores declaran no tener conflictos de interés en la publicación de este artículo.

\section{REFERENCIAS BIBLIOGRÁFICAS}

1. Chippaux JP. Snake-bites: appraisal of the global situation. Bull World Health Organ. 1998;76(5):515-24.

2. Zavaleta A. Mordedura de serpiente (ofidismo): un problema de salud en el Perú. Rev Med Hered. 2004;15(2):61-63.

3. Fuly AL, Francischetti IM, Zingali RB, Carlini CR. Partial purification and some physicochemical properties of phospholipases A2 from the venom of the bushmaster snake (Lachesis muta). Braz J Med Biol Res. 1993;26(5):459-63.

4. Rucavado A, Flores-Sanchez E, Franceschi A, Magalhaes A, Gutierrez JM. Characterization of the local tissue damage induced by LHF-II, a metalloproteinase with weak hemorrhagic activity isolated from Lachesis muta muta snake venom. Toxicon. 1999;37(9):1297-312.

5. Lizano S, Lambeau G, Lazdunski M. Cloning and cDNA sequence analysis of Lys(49) and Asp(49) basic phospholipase $\mathrm{A}(2)$ myotoxin isoforms from Bothrops asper. Int J Biochem Cell Biol. 2001;33(2):127-32.

6. Schwede T, Kopp J, Guex N, Peitsch MC. SWISSMODEL: An automated protein homology-modeling server. Nucleic Acids Res. 2003;31(13):3381-85.

7. Han SK, Yoon ET, Scott DL, Sigler PB, Cho W. Structural aspects of interfacial adsorption. A crystallographic and site-directed mutagenesis study of the phospholipase A2 from the venom of Agkistrodon piscivorus piscivorus. J Biol Chem. 1997;272(6):3573-82.

8. Deshimaru M, Ogawa T, Nakashima K, Nobuhisa I, Chijiwa T, Shimohigashi Y, et al. Accelerated evolution of crotalinae snake venom gland serine proteases. FEBS Lett. 1996;397(1):83-88.

9. Gibbs HL, Rossiter W. Rapid evolution by positive selection and gene gain and loss: PLA(2) venom genes in closely related Sistrurus rattlesnakes with divergent diets. J Mol Evol. 2008;66(2):151-66.

10. Nobuhisa I, Nakashima K, Deshimaru M, Ogawa T, Shimohigashi Y, Fukumaki Y, et al. Accelerated evolution of Trimeresurus okinavensis venom gland phospholipase A2 isozyme-encoding genes. Gene. 1996;172(2):267-72.

11. Ohno M, Chijiwa T, Oda-Ueda N, Ogawa T, Hattori S. Molecular evolution of myotoxic phospholipases A2 from snake venom. Toxicon. 2003;42(8):841-54.

12. Murakami MT, Arruda EZ, Melo PA, Martinez AB, CalilElias S, Tomaz MA, et al. Inhibition of myotoxic activity of Bothrops asper myotoxin II by the anti-trypanosomal drug suramin. J Mol Biol. 2005;350(3):416-26.

13. Patlak M. From viper's venom to drug design: treating hypertension. FASEB J. 2004;18(3):421.

14. Singh N, Jabeen T, Sharma S, Somvanshi RK, Dey S, Srinivasan A, et al. Specific binding of non-steroidal anti-inflammatory drugs (NSAIDs) to phospholipase A2: structure of the complex formed between phospholipase A2 and diclofenac at 2.7 A resolution. Acta Crystallogr D Biol Crystallogr. 2006;62(Pt 4):410-16. 
15. Rigoni M, Paoli M, Milanesi E, Caccin P, Rasola A, Bernardi $\mathbf{P}$, et al. Snake phospholipase A2 neurotoxins enter neurons, bind specifically to mitochondria, and open their transition pores. J Biol Chem. 2008;283(49):3401320.

16. Montecucco C, Gutierrez JM, Lomonte B. Cellular pathology induced by snake venom phospholipase A2 myotoxins and neurotoxins: common aspects of their mechanisms of action. Cell Mol Life Sci. 2008;65(18):2897912.

17. Sim KL. Purification and preliminary characterisation of praelongin phospholipases, antiplatelet agents from the snake venom of Acanthophis praelongus. Biochim Biophys Acta. 1998;1379(2):198-206.

18. Fuly AL, De Miranda AL, Zingali RB, Guimaraes JA. Purification and characterization of a phospholipase A2 isoenzyme isolated from Lachesis muta snake venom. Biochem Pharmacol. 2002;63(9):1589-97.

19. Damico DC, Bueno LG, Rodrigues-Simioni L, Marangoni S, Da Cruz-Hofling MA, Novello JC. Functional characterization of a basic D49 phospholipase A2 (LmTX-I) from the venom of the snake Lachesis muta muta (bushmaster). Toxicon. 2006;47(7):759-65.

20. Sanz L, Escolano J, Ferretti M, Biscoglio MJ, Rivera E, Crescenti EJ, et al. Snake venomics of the South and Central American bushmasters. Comparison of the toxin composition of Lachesis muta gathered from proteomic versus transcriptomic analysis. J Proteomics. 2008;71(1):46-60.

21. Damico DC, Lilla S, de Nucci G, Ponce-Soto LA, Winck FV, Novello JC, et al. Biochemical and enzymatic characterization of two basic Asp49 phospholipase A2 isoforms from Lachesis muta muta (Surucucu) venom. Biochim Biophys Acta. 2005;1726(1):75-86.

22. Li Y, Yu BZ, Zhu H, Jain MK, Tsai MD. Phospholipase A2 engineering. Structural and functional roles of the highly conserved active site residue aspartate-49. Biochemistry. 1994;33(49):14714-22.

23. Lomonte B, Angulo Y, Calderon L. An overview of lysine-49 phospholipase A2 myotoxins from crotalid snake venoms and their structural determinants of myotoxic action. Toxicon. 2003;42(8):885-901.

24. Scott DL, White SP, Otwinowski Z, Yuan W, Gelb MH, Sigler PB. Interfacial catalysis: the mechanism of phospholipase A2. Science. 1990;250(4987):1541-6.

25. Pungercar J, Krizaj I. Understanding the molecular mechanism underlying the presynaptic toxicity of secreted phospholipases A2. Toxicon. 2007;50(7):871-92.

26. Rowan EG. What does beta-bungarotoxin do at the neuromuscular junction? Toxicon. 2001;39(1):107-18.

27. Tsai IH, Wang YM, Chen YH, Tsai TS, Tu MC. Venom phospholipases A2 of bamboo viper (Trimeresurus stejnegeri): molecular characterization, geographic variations and evidence of multiple ancestries. Biochem J. 2004;377(Pt 1):215-23.

28. Fuly AL, Calil-Elias S, Zingali RB, Guimaraes JA, Melo PA. Myotoxic activity of an acidic phospholipase A2 isolated from Lachesis muta (Bushmaster) snake venom. Toxicon. 2000;38(7):961-72.
29. de Albuquerque Modesto JC, Spencer PJ, Fritzen M, Valenca RC, Oliva ML, da Silva MB, et al. BE-I-PLA2, a novel acidic phospholipase A2 from Bothrops erythromelas venom: isolation, cloning and characterization as potent anti-platelet and inductor of prostaglandin 12 release by endothelial cells. Biochem Pharmacol. 2006;72(3):377-84.

30. Rodrigues RS, Izidoro LF, Teixeira SS, Silveira LB, Hamaguchi A, Homsi-Brandeburgo MI, et al. Isolation and functional characterization of a new myotoxic acidic phospholipase $\mathrm{A}(2)$ from Bothrops pauloensis snake venom. Toxicon. 2007;50(1):153-65.

31. Roberto PG, Kashima S, Soares AM, Chioato L, Faca VM, Fuly AL, et al. Cloning and expression of an acidic platelet aggregation inhibitor phospholipase A2 cDNA from Bothrops jararacussu venom gland. Protein Expr Purif. 2004;37(1):102-8.

32. Liu $\mathbf{X}$, Wu $\mathbf{X}$, Zhou $\mathbf{Y}$. Identification of key residues responsible for enzymatic and platelet-aggregationinhibiting activities of acidic phospholipase A2S from Agkistrodon halys Pallas. J Nat Toxins. 2001;10(1):43-55.

33. Perumal Samy R, Pachiappan A, Gopalakrishnakone $P$, Thwin MM, Hian YE, Chow VT, et al. In vitro antimicrobial activity of natural toxins and animal venoms tested against Burkholderia pseudomallei. BMC Infect Dis. 2006;6:100.

34. Kordis D, Bdolah A, Gubensek F. Positive Darwinian selection in Vipera palaestinae phospholipase A2 genes is unexpectedly limited to the third exon. Biochem Biophys Res Commun. 1998;251(2):613-19.

35. Valiente C, Moreno E, Sittenfeld A, Lomonte B, Gutierrez JM. An electrophoretic study on phospholipase A2 isoenzymes in the venoms of Central American crotaline snakes. Toxicon. 1992;30(8):815-23.

36. Chijiwa T, Hamai S, Tsubouchi S, Ogawa T, Deshimaru $\mathbf{M}$, Oda-Ueda $\mathbf{N}$, et al. Interisland mutation of a novel phospholipase A2 from Trimeresurus flavoviridis venom and evolution of Crotalinae group II phospholipases A2. J Mol Evol. 2003;57(5):546-54.

37. Creer S, Malhotra A, Thorpe RS, Stocklin RS, Favreau PS, Hao Chou WS. Genetic and ecological correlates of intraspecific variation in pitviper venom composition detected using matrix-assisted laser desorption time-offlight mass spectrometry (MALDI-TOF-MS) and isoelectric focusing. J Mol Evol. 2003;56(3):317-29.

38. Nakashima K, Nobuhisa I, Deshimaru M, Nakai M, Ogawa T, Shimohigashi Y, et al. Accelerated evolution in the protein-coding regions is universal in crotalinae snake venom gland phospholipase A2 isozyme genes. Proc Natl Acad Sci U S A. 1995;92(12):5605-9.

39. Ogawa T, Kitajima M, Nakashima K, Sakaki Y, Ohno M. Molecular evolution of group II phospholipases A2. J Mol Evol. 1995;41(6):867-77.

Correspondencia: Karim L. Jimenez

Dirección: calle César Gonzales, XXX 9,1 A, Madrid-28027, España

Teléfono: (034)-911536498

Correo electrónico: karimlizeth@gmail.com. 